\title{
Resolution Enhancement by Prediction of the High-Frequency Image Based on the Laplacian Pyramid
}

\author{
Bo-Won Jeon, ${ }^{1}$ Rae-Hong Park, ${ }^{1}$ and Seungjoon Yang ${ }^{2}$ \\ ${ }^{1}$ Department of Electronic Engineering, School of Engineering, Sogang University, C.P.O. Box 1142, Seoul 100-611, Korea \\ ${ }^{2}$ Digital Media Research and Development Center, Samsung Electronics Corporation, Ltd., Suwon 442-742, Korea
}

Received 30 November 2004; Revised 20 March 2005; Accepted 7 April 2005

\begin{abstract}
According to recent advances in digital image processing techniques, interest in high-quality images has been increased. This paper presents a resolution enhancement (RE) algorithm based on the pyramid structure, in which Laplacian histogram matching is utilized for high-frequency image prediction. The conventional RE algorithms yield blurring near-edge boundaries, degrading image details. In order to overcome this drawback, we estimate an HF image that is needed for RE by utilizing the characteristics of the Laplacian images, in which the normalized histogram of the Laplacian image is fitted to the Laplacian probability density function (pdf), and the parameter of the Laplacian pdf is estimated based on the Laplacian image pyramid. Also, we employ a control function to remove overshoot artifacts in reconstructed images. Experiments with several test images show the effectiveness of the proposed algorithm.
\end{abstract}

Copyright @ 2006 Hindawi Publishing Corporation. All rights reserved.

\section{INTRODUCTION}

In most electronic imaging applications, resolution enhancement (RE) of images containing high-density pixels with high detail is desired and often required [1]. Most of all, fast advance in multimedia technology requires RE more and more. One application is to reconstruct a higher-quality digital image from a low-resolution (LR) image that is obtained with an inexpensive camera/camcorder for printing or frame-freeze purpose. Another application is conversion from a National Television System Committee (NTSC) video signal to a high-definition television (HDTV) signal to display a standard video signal on HDTV with less visual artifacts. Also, synthetic zooming of the region of interest (ROI) is another important application in TV home shopping. Generally, there are several issues to be considered in RE: unavoidable blurring artifacts, reconstruction of HF details with annoying artifacts, and high computational cost. When a portion of a digital image acquired once is enlarged for display, RE or enlargement is an indispensable digital image processing technique. RE of digital images corresponds to reduction of the spatial sampling interval, in which HF components of the resolution-enhanced images become larger. Therefore, for effective RE of digital images, it is necessary to estimate by some means the HF components that are lost in image data acquisition.
Conventional linear interpolation schemes (e.g., bilinear and bicubic) based on space-invariant models produce interpolated images with blurred edges and annoying artifacts $[2,3]$. Linear interpolation is generally preferred for the computational simplicity, not for the performance.

Many algorithms [4-7] have been proposed to improve the subjective quality of the interpolated images by imposing more accurate image models. Li and Orchard proposed an edge-directed interpolation method that is based on the geometric duality between covariances of LR and HR images [4]. The edge-directed property of covariance-based adaptation gives the capability of tuning the interpolation coefficients to match an arbitrarily oriented step edge. This method demonstrates significant improvements on visual quality over linear interpolation methods, but a computational cost is high. Biancardi et al. proposed an image magnification method [5]. The idea underlying this work is to estimate the phases and frequencies of lost waveforms from the original LR image and then to synthesize them in the HR image. Their technique takes advantage of subpixel edge estimation from the LR image to direct the subsequent polynomial interpolation step. Also, Leu presented an approach that can maintain both the continuity and sharpness of edges when enlarging an image based on a ramp edge model [6]. This method first locates all the edge pixels in a given image, extracts five parameters, uses them to generate the edges, and finally employs 


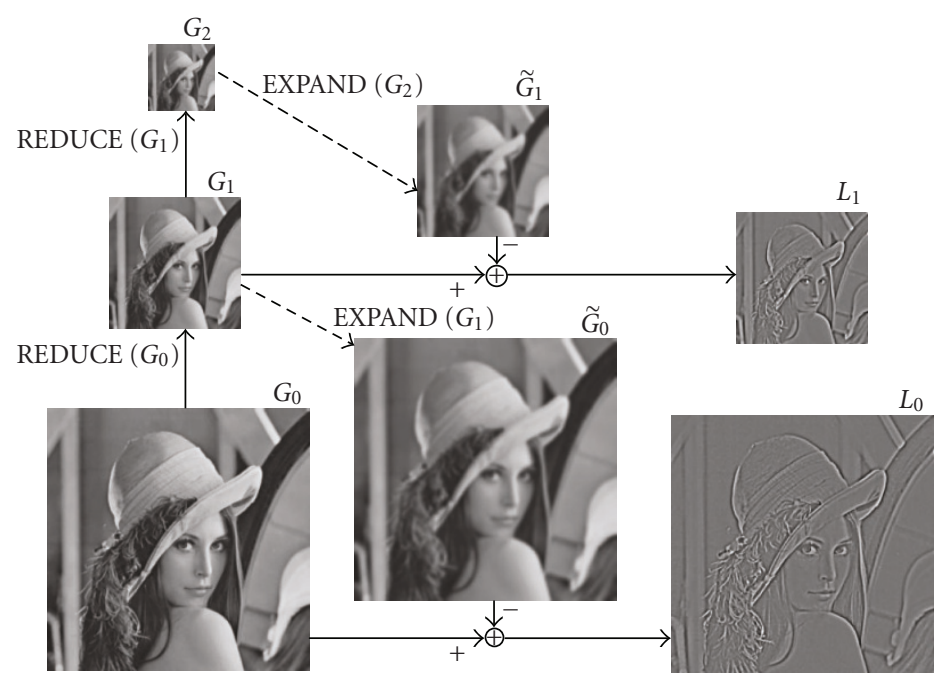

Figure 1: Gaussian/Laplacian image pyramid $(N=3)$.

bilinear interpolation to enlarge nonedge areas. Recently, Wang and Ward proposed an orientation-guided interpolation method, in which image contours are divided into edges and ridges, and employed directional interpolation based on the estimated orientation of edges and ridges [7]. In Greenspan et al.'s method [8], RE of images is treated as a prediction problem of unknown HF Laplacian components within the framework of the Laplacian-pyramid-layered representation, in which an HR image is predicted from the LR image by implicitly using the following characteristics: strong edges and details of images are confirmed as zero crossings at the same location in several adjacent layers of the Laplacian pyramid. Also, Takahashi and Taguchi proposed an RE method [9] that is based on Greenspan et al.'s method [8], in which the enlargement ratio is arbitrary with two constant parameters. Carey et al. proposed a wavelet-based interpolation method that imposes no continuity constraints [10]. This algorithm estimates the regularity of edges by measuring the decay of wavelet transform coefficients across scales and preserves the underlying regularity by extrapolating a new subband that is to be used in image synthesis. In addition, super-resolution methods based on a set of training images were proposed by Freeman et al. [11] and Sun et al. [12]. These methods estimate missing HR detail that is not present in the LR image and cannot be made visible by simple sharpening. Their results maintain sharpness of edges and lines as well as texture details.

In this paper, an RE method is proposed by predicting an HR Laplacian image from an LR image. It predicts the HF image based on the Gaussian/Laplacian pyramid structure, as in Greenspan et al.'s method. Utilizing characteristics of the Laplacian images, we estimate an HF image that is needed for RE. In addition, we employ a control function to remove overshoot artifacts in reconstructed images.

The rest of the paper is organized as follows. In Section 2, Gaussian/Laplacian pyramid construction is briefly reviewed. Also parameter estimation and control function design for prediction of an HF image are presented. Experimental results and discussions are shown in Section 3. Finally, conclusions are given in Section 4.

\section{PROPOSED RE ALGORITHM BASED ON THE LAPLACIAN PYRAMID}

The proposed RE algorithm predicts an HF image based on the Laplacian pyramid structure, where the Laplacian pyramid represents a set of images with HF components. We predict the HF image by utilizing statistical characteristics of the Laplacian images, in which the normalized histogram of the Laplacian image data is fitted to the Laplacian probability density function (pdf) and the parameter of the Laplacian pdf is estimated based on the Laplacian pyramid images. In addition, we employ a control function to remove overshoot artifacts in reconstructed images. Generally, high-frequency components of natural images can be modeled as generalized Gaussian distribution (GGD). Note that the Laplacian distribution has a single parameter, whereas the GGD has two parameters to determine. So, we prefer the Laplacian distribution in fitting the distribution of the high-frequency image (see [13]).

\subsection{Gaussian/Laplacian image pyramid}

The Gaussian pyramid is a set of LR images obtained by lowpass filtering and then decimation. The Laplacian pyramid is defined by the difference image between two Gaussian images at adjacent levels, containing HF image components such as edge details in an image. Figure 1 shows construction of Gaussian/Laplacian pyramids, in which lowpass filtering is applied to a given resolution image and then decimation follows to get a lower-resolution image [13].

Gaussian pyramid construction can be described as follows. Let $G_{n}$ be the $n$th Gaussian image of the input image 


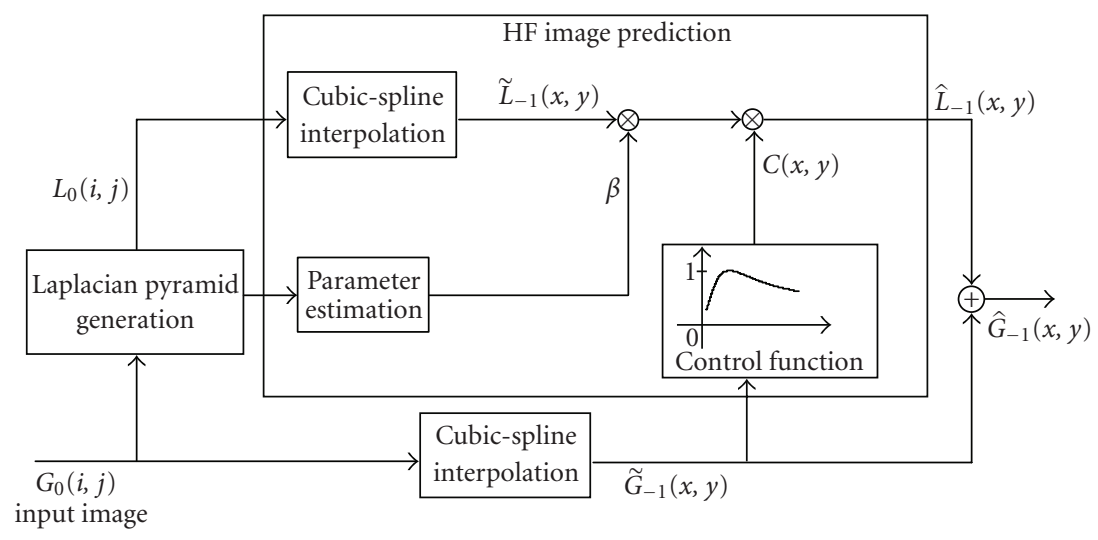

FIGURE 2: Block diagram of the proposed RE algorithm.

$G_{0}$, then $G_{n+1}$ is constructed by a $\operatorname{REDUCE}(\cdot)$ operator:

$$
G_{n+1}=\operatorname{REDUCE}\left(G_{n}\right), \quad 0 \leq n \leq N-1,
$$

where $N$ signifies the total number of levels in the pyramid structure, with $G_{0}$ denoting the highest resolution. Let $\tilde{G}_{n}$ be the image expanded from $G_{n+1}$ by an $\operatorname{EXPAND~(\cdot )~operator:~}$

$$
\tilde{G}_{n}=\operatorname{EXPAND}\left(G_{n+1}\right), \quad 0 \leq n \leq N-1 .
$$

The Laplacian pyramid is generated by

$$
L_{n}=\tilde{G}_{n}-\operatorname{EXPAND}\left(G_{n+1}\right), \quad 0 \leq n \leq N-1,
$$

where $L_{n}$ denotes the $n$th Laplacian pyramid image of $G_{0}$. The interesting reader is referred to [13] for details of the construction of the Gaussian/Laplacian pyramid.

In this paper, we make use of the statistical property of the Laplacian image in predicting the HF image, noting that the variance increases as the pyramid level $n$ increases. For example, the variance of $L_{1}$ image is larger than that of $L_{0}$ image.

\subsection{Overview of the proposed RE algorithm}

Conventional RE algorithms show blurring near-edge boundaries, and thus cannot effectively restore HF components lost in digitization of the image. To overcome this drawback, our objective is to acquire an HR image by predicting the HF image from the Laplacian pyramid.

The Laplacian pyramid represents a set of bandpass/highpass images, from which we can estimate the HF image needed for RE by utilizing statistical characteristics of the Laplacian pyramid images, in which the normalized histogram of the Laplacian image data is fitted to the Laplacian pdf and the parameter of the Laplacian pdf is estimated based on the Laplacian pyramid images. Figure 2 shows the block diagram of the proposed algorithm, in which $G_{0}(i, j)$ denotes a given LR input image and $\widehat{G}_{-1}(x, y)$ represents an HR output image to be constructed. Prediction of the HF image $\widehat{L}_{-1}(x, y)$ requires three inputs: $\tilde{L}_{-1}(x, y)$ obtained by initial (cubic-spline) interpolation of $L_{0}(i, j)$, an estimated scaling constant $\beta$, and the control function $C(x, y)$.
The output $\hat{G}_{-1}(x, y)$ is obtained by adding the predicted HF image $\widehat{L}_{-1}(x, y)$ to the initially (cubic-spline) interpolated image $\tilde{G}_{-1}(x, y)$ :

$$
\hat{G}_{-1}(x, y)=\tilde{G}_{-1}(x, y)+\widehat{L}_{-1}(x, y)
$$

where $\hat{L}_{-1}(x, y)$ is predicted by

$$
\widehat{L}_{-1}(x, y)=C(x, y) \beta \tilde{L}_{-1}(x, y) .
$$

Generating the new output image $\hat{G}_{-1}(x, y)$ entails predicting $\widehat{L}_{-1}(x, y)$ image as the HF image by pyramid representation (see (3)). Because we do not know $\widehat{G}_{-1}$ image, there is no choice but to generate $\tilde{L}_{-1}(x, y)$ image by initial guess. In this paper, we employ cubic-spline interpolation method for its performance and computational load to construct $\tilde{L}_{-1}(x, y)$ image.

\subsection{Prediction of the HF image}

This section describes estimation of the scaling parameter $\beta$ that is a global constant needed for prediction of the HF image. The constant $\beta$ is estimated by least-squares line fitting of parameter pairs of Laplacian pdf fitting, in which Laplacian pdf fitting is based on statistical characteristics of the Laplacian image: the normalized histogram of the Laplacian image data is assumed to follow the Laplacian pdf. Note that the variance increases as the pyramid level $n$ increases.

\subsubsection{Laplacian fitting}

The Laplacian pdf $f_{P}(p)$ is expressed as

$$
f_{P}(p)=\frac{\alpha}{2} e^{-\alpha|p|},
$$

where $p$ denotes the pixel value of the Laplacian image with zero mean and variance $\sigma^{2}=2 / \alpha^{2}$. Fitting the given Laplacian image data to the Laplacian pdf yields the parameter $\alpha_{n}$ as follows:

$$
\alpha_{n}=\frac{\sqrt{2}}{\sigma_{n}}, \quad \sigma_{n}^{2}=\sum_{l=-\infty}^{l=+\infty}\left(l-\mu_{n}\right)^{2} h(l),
$$




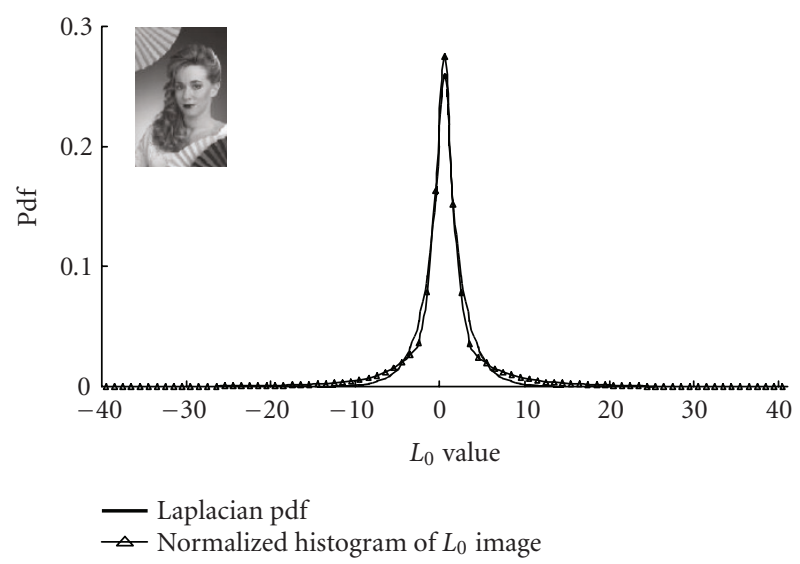

Figure 3: Laplacian pdf fitting $\left(512 \times 768 L_{0}\right.$ image, $\left.\alpha_{0}=0.266, \mu_{0}=0.147\right)$.

where $n$ signifies the level of the pyramid and $h(l)$ signifies the normalized histogram for pixel value $l$ with mean $\mu_{n}$ and variance $\sigma_{n}^{2}$.

Figure 3 shows an example of Laplacian pdf fitting of $L_{0}$ image, giving $\alpha_{0}=0.266$ and $\mu_{0}=0.147$, where the $512 \times 768$ Woman image with $n=0$ is used. The Laplacian image is approximately fitted to the Laplacian pdf. Similarly, $\alpha_{n}, 1 \leq$ $n \leq 4$, are computed.

Such computed $\alpha_{n}$ are used to explain the statistical relationship between the LR and HR images. Using a histogrammatching method, we can write this relationship between two pdfs of Laplacian images at adjacent pyramid levels as

$$
\frac{1}{2}+\int_{0}^{p} \frac{\alpha_{n}}{2} e^{-\alpha_{n} u} d u=\frac{1}{2}+\int_{0}^{q} \frac{\alpha_{n-1}}{2} e^{-\alpha_{n-1} v} d v,
$$

which leads to

$$
q=\frac{\alpha_{n}}{\alpha_{n-1}} p
$$

where $p$ and $q$ denote LR and HR pixel values, respectively. Applying (9) to the HF image prediction gives

$$
\widehat{L}_{-1}(x, y)=C(x, y) \frac{\alpha_{0}}{\hat{\alpha}_{-1}} \tilde{L}_{-1}(x, y)=C(x, y) \beta \tilde{L}_{-1}(x, y),
$$

where $\beta$ is a scaling constant and $\tilde{L}_{-1}$ denotes the initially interpolated image of $L_{0}$. Here, $\hat{\alpha}_{-1}$ is an unknown constant that we want to estimate. If $\hat{\alpha}_{-1}$ is estimated, we use (10) to predict HF image, which is then added to the initially interpolated image $\left(\tilde{G}_{-1}\right)$ of $G_{0}$ to yield $\hat{G}_{-1}$.

\subsubsection{Estimation of the scaling constant $\beta$}

The scaling constant $\beta=\alpha_{0} / \hat{\alpha}_{-1}$ is used for prediction of HF image $\widehat{L}_{-1}$, where $\hat{\alpha}_{-1}$ is estimated by least-squares line fitting, using $(N-1)$ sets of $\left(\alpha_{n}, \alpha_{n-1}\right), 1 \leq n \leq N-1$, computed from the Laplacian pyramid images. Least-squares line fitting is expressed as

$$
\mathbf{H}=\left(\mathbf{A}^{\mathrm{T}} \mathbf{A}\right)^{-1}\left(\mathbf{A}^{\mathrm{T}} \mathbf{V}\right),
$$

where $\mathbf{Y}, \mathbf{A}$, and $\mathbf{X}$ are given by

$$
\mathbf{V}=\left[\begin{array}{c}
\alpha_{0} \\
\vdots \\
\alpha_{N-2}
\end{array}\right], \quad \mathbf{A}=\left[\begin{array}{cc}
\alpha_{1} & 1 \\
\vdots & \vdots \\
\alpha_{N-1} & 1
\end{array}\right], \quad \mathbf{H}=\left[\begin{array}{l}
a \\
b
\end{array}\right]
$$

with $a$ and $b$ denoting a slope and an intersection at the vertical axis of the fitted line, respectively. Then, $\hat{\alpha}_{-1}$ is estimated by linear regression:

$$
\hat{\alpha}_{-1}=a \alpha_{0}+b .
$$

Figure 4 shows an example of least-squares line fitting, giving $a=0.898$ and $b=0.054$ for the $512 \times 768$ Woman image with $N=5$. Finally, using the estimated scaling constant $\beta$, we can predict HF image $\widehat{L}_{-1}$ for RE.

\subsection{Control function design}

We explained how to estimate the scaling constant $\beta$ based on the Laplacian pyramid, which will be used for prediction of HF image $\widehat{L}_{-1}$. The scaling constant $\beta$ is applied to all pixels in $\tilde{L}_{-1}$, that is, $\beta$ can be regarded as the global constant that determines the final image quality and does not reflect local characteristic of the image.

As shown in Figure 2, $\tilde{G}_{-1}(x, y)$ is used to formulate a control function $C(x, y)$. If $\beta$ alone is applied to prediction of HF image, we observe two types of artifacts in $\hat{G}_{-1}$ : noise amplification over smooth regions and overshoots near-edge regions. The former can be explained from the fact that the RE method based on HF image prediction puts an emphasis on even small variations. On the contrary, abrupt luminance changes in the input image can produce overshoots, which is explained by the human visual system through the Mach band effect [2]. In Takahashi and Taguchi's method [9], the HF components used in Greenspan et al.'s method [8] are extracted from a half-band filter and the resulting image $\widehat{L}_{-1}$ is used for RE. We design the control function to reduce distortions, or equivalently to define the local scaling function 


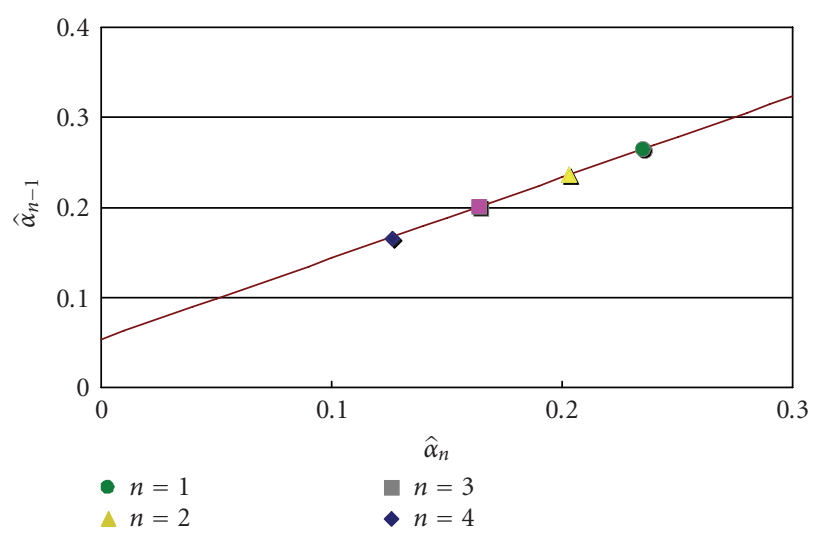

FIGURE 4: Estimation of parameter $\alpha_{-1}$ by least-squares line fitting $(a=0.898, b=0.054)$.

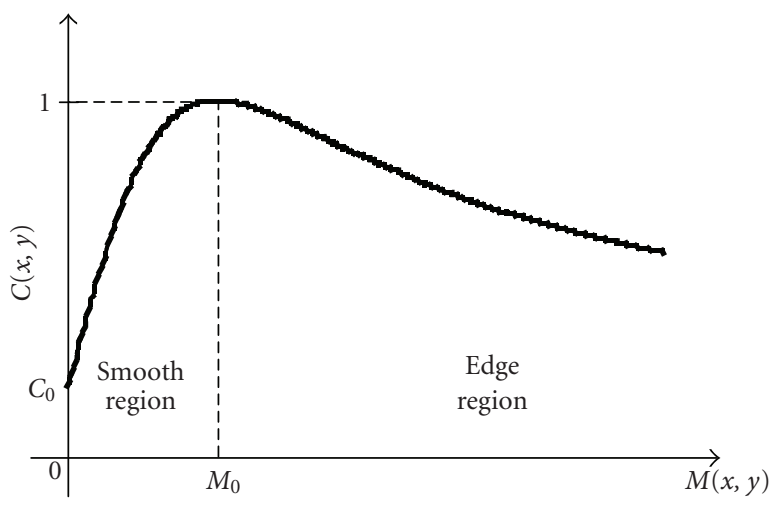

Figure 5: Control function $C(x, y)$ as a function of the local activity $M(x, y)$.

$\tilde{\beta}(x, y)$, which reflects the local activity of an input image, rather than the global constant $\beta$, that is, (5) is modified as

$$
\widehat{L}_{-1}(x, y)=\tilde{\beta}(x, y) \tilde{L}_{-1}(x, y),
$$

where $\tilde{\beta}(x, y)=\beta C(x, y)$ denotes the scaling function that reflects the local activity.

Figure 5 shows the control function as a function of the local activity $M(x, y)$, with $M_{0}$ and $c_{0}$ denoting parameters that adjust the control function. As a local activity $M(x, y)$, we use the magnitude of the Sobel operator that reflects the intensity variations on the local region. Observation shows that overshoot artifacts are introduced near-edge points, where the magnitude of the Sobel operator is large. So, if we use the magnitude of the Sobel operator as a local activity, overshoot artifacts near-edge regions can be reduced. Two constant parameters are employed in $C(x, y): M_{0}$ signifies a decision boundary value that classifies each pixel into the pixel in smooth or edge regions and $c_{0}$ represents a value of the control function $C(x, y)$ when $M(x, y)$ is 0 . The constant $M_{0}$ is employed to reduce overshoots near-edge regions, whereas $c_{0}$ is used to reduce the noise amplification over smooth regions. Note that the control function $C(x, y)$ satisfies

$$
\lim _{M(x, y) \rightarrow 0} C(x, y)=c_{0}, \quad \lim _{M(x, y) \rightarrow \infty} C(x, y)=0 .
$$

Ramponi and Polesel proposed rational filters for unsharp masking [14], where the filter output depends on the local activity. Also, Cheikh and Gabbouj [15] proposed a rational unsharp masking filter that limits the overshoots near sharp edges and attenuates noise in flat areas. The characteristics of the control function used in the proposed algorithm are similar to those of the rational filter by Ramponi and Polesel [14]. The rational function $C(x, y)$ used in our study to reflect the local activity of the image is defined as

$$
C(x, y)=\frac{M(x, y)+b}{k(M(x, y))^{2}+h},
$$

where $b, h$, and $k$ are given by

$$
b=\frac{c_{0} M_{0}}{2\left(1-c_{0}\right)}, \quad h=\frac{M_{0}}{2\left(1-c_{0}\right)}, \quad k=\frac{1}{2 M_{0}},
$$

which are easily checked with the fact that the maximum value of $C(x, y)$ is equal to 1 . Employing such a function, 


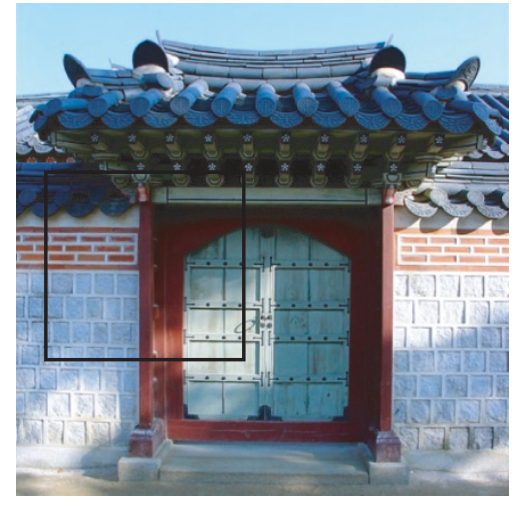

(a)

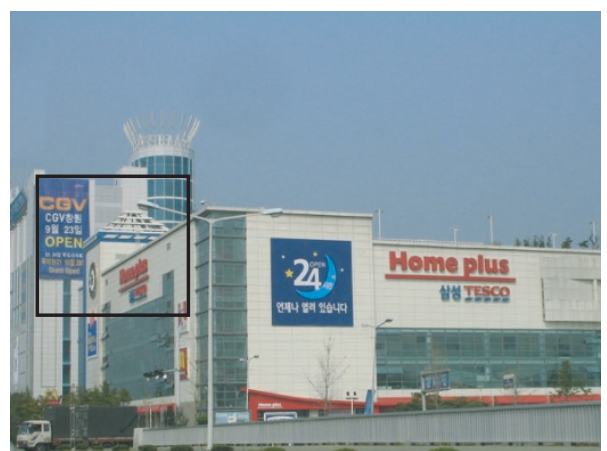

(b)

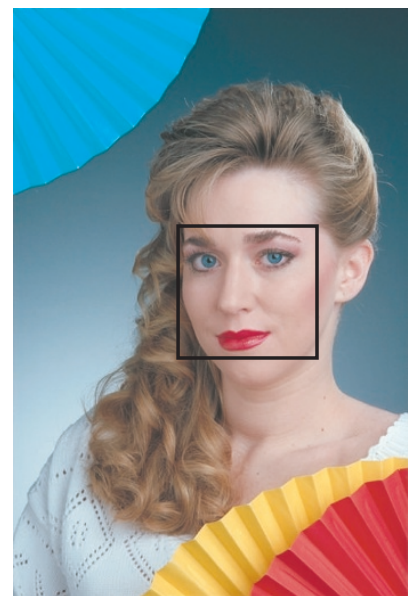

(c)

Figure 6: Test images: (a) Gate image $(512 \times 512)$, (b) Mart image $(640 \times 480)$, and (c) Woman image $(512 \times 768)$.

selected details having low and medium sharpness are enhanced, whereas amplification of small variations is very limited. Steep edges, which do not need further emphasis, remain almost unaffected.

\section{EXPERIMENTAL RESULTS AND DISCUSSIONS}

In this section, we present experimental results. As explained previously, the predicted HF image using the global constant $\beta$ and the control function $C(x, y)$ considering the local activity is added to the interpolated input image, in which three parameters $\left(\beta, M_{0}\right.$, and $\left.c_{0}\right)$ are used. The scaling constant $\beta$ is estimated by least-squares fitting of pdfs embedded in the Laplacian pyramid structure. The constant $c_{0}$ is related to suppression of amplification of small variations (e.g., noise components) in smooth regions. Thus, $c_{0}$ must be small: in all experiments, $c_{0}$ is set to zero, which gives the control function in (16) as a function of a single parameter $M_{0}$, yielding $b_{0}=0, h=M_{0} / 2$, and $k=1 /\left(2 M_{0}\right)$. With $c_{0}=0$, smooth components of $\tilde{G}_{-1}$ are directly transferred to $\hat{G}_{-1}$. The performance of four RE methods (cubic-spline interpolation, Li and Orchard's method, Greenspan et al's method, and the proposed method) is compared. In practical cases of interpolation, the original HR image is not given, thus image quality of interpolated images is subjectively compared. The significant point for performance comparison based on subjective image quality is sharpness and connectivity of edges. Note that the proposed RE algorithm adds the predicted HF image $\widehat{L}_{-1}$ to the initially interpolated image $\tilde{G}_{-1}$ by cubic-spline, yielding a visually enhanced output $\hat{G}_{-1}$.

Test images used in experiments are RGB color images. We convert RGB color format to YUV format. Then, only Y (luminance) component is processed by the RE algorithms, and $\mathrm{U}$ and $\mathrm{V}$ components are magnified by bilinear interpolation. Since the human visual system is sensitive to luminance components and relatively insensitive to color components, magnifying $\mathrm{U}$ and $\mathrm{V}$ components by a simple bilinear interpolation method has not influence on image quality much. Thus, gray scale images (Y components) are shown to show the performance of the proposed RE algorithm. In all experiments, test images are magnified by a factor of two horizontally and vertically.

The proposed algorithm has been tested using a number of different images and three examples are shown in this section. First, we explain characteristics of test images and determination of parameter values. Then, we compare the performance of four RE algorithms. Finally, we present the computational cost and the peak signal-to-noise ratio (PSNR) of the interpolation algorithms.

\subsection{Test images}

In experiments, we show three test images: Gate, Mart, and Woman images, as shown in Figure 6. The $512 \times 512$ Gate image in Figure 6(a) has strong edge components as well as flat regions. In the first experiments with the Gate image, three different $M_{0}$ values are used to show the effect of parameter $M_{0}$ on the final performance of interpolation. As claimed previously, the values of two parameters $\left(c_{0}\right.$ and $\left.M_{0}\right)$ are not critical. In experiments with three test images, $c_{0}$ is fixed. The $640 \times 480$ Mart image in Figure 6(b) contains relatively smooth regions as well as regions with abrupt changes. This image contains the character region. In the second experiment, we show the enlarged character region to show the effectiveness of the proposed algorithm, where $M_{0}$ is set to 40 . The $512 \times 768$ Woman image in Figure 6(c) has strong step edges, thus in the third experiment, $M_{0}$ is set to 50 . Note that the Woman image contains abrupt luminance changes.

\subsection{Experimental results}

As claimed previously, in experiments we select parameter $M_{0}$ that gives good results (to reduce overshoot artifacts near 


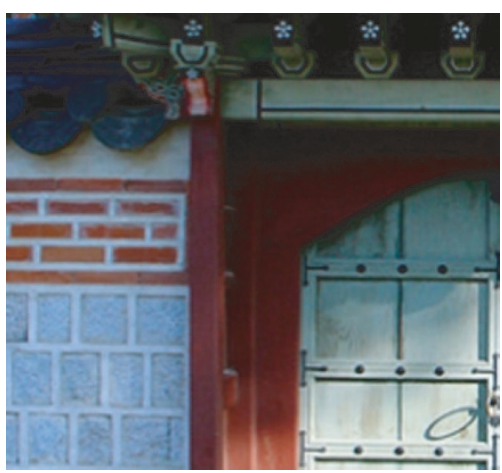

(a)

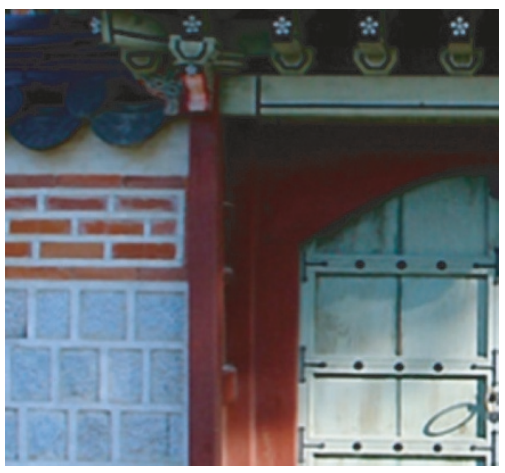

(d)

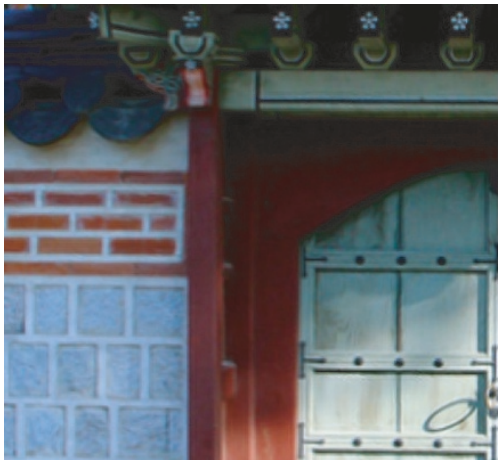

(b)

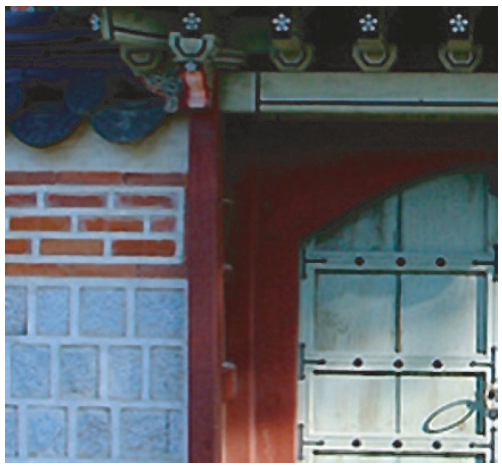

(e)

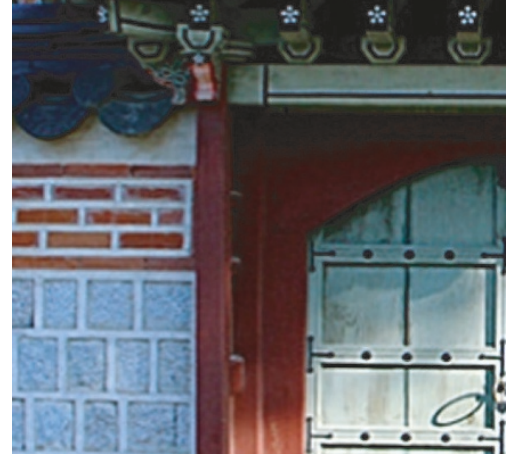

(c)

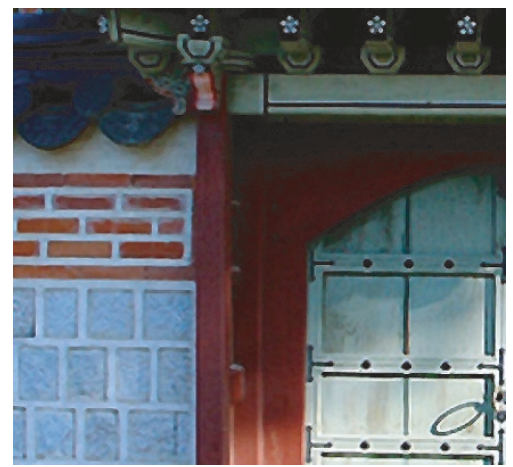

(f)

FIgURe 7: Comparison of RE results $(1024 \times 1024$ Gate image, $200 \%$ zoomed part: $340 \times 340)$ : (a) cubic-spline interpolation, (b) Li and Orchard's method, (c) Greenspan et al.'s method, (d) proposed algorithm $\left(M_{0}=35, c_{0}=0.0\right)$, (e) proposed algorithm $\left(M_{0}=55, c_{0}=0.0\right)$, and (f) proposed algorithm $\left(M_{0}=80, c_{0}=0.0\right)$.

edge regions). If an image contains strong edge regions, we set $M_{0}$ value to a large value (e.g., 50), otherwise to a small value. Figures 7, 8, and 9 show portions of images, indicated by boxes on the original test image in Figures 6(a), 6(b), and $6(\mathrm{c})$, respectively, which are zoomed by a factor two horizontally and vertically.

Figures 7(a)-7(c) show $340 \times 340$ zoomed images that are portions of the image in Figure 6(a), interpolated by cubicspline interpolation, Li and Orchard's method, Greenspan et al.'s method, respectively. Figures 7(d)-7(f) show those interpolated by the proposed algorithm with three different values of $M_{0}: 35,55$, and 80 , respectively. The Gate image has strong edges near boundaries of the wall and gate. Figures $7(d)-$ $7(\mathrm{f})$ show the effect of $M_{0}$ value, in which Figure 7(e) shows the optimal result $\left(M_{0}=55\right)$. If $M_{0}$ is small (large), overshoot artifacts become unnoticeable (remarkable), for example, if $M_{0}$ is larger than 55, overshoot artifacts are visible as shown in Figure 7(f). The result of Greenspan et al.'s method shows unnecessary changes in smooth regions, whereas that of Li and Orchard's method shows blurring while edge connectivity is good. The result of the proposed method with $M_{0}=55$ preserves edge connectivity well, as in Li and Orchard's method. Faithful edge preservation based on edge connectivity requires edge direction as well as edge magnitude, which is beyond the scope of this paper. Ensuring edge connectivity is to be investigated as future work.

Figures 8 (a) -8 (d) show $400 \times 280$ zoomed images that are portions of the image in Figure 6(b), interpolated by cubicspline interpolation, $\mathrm{Li}$ and Orchard's method, Greenspan et al.'s method, and the proposed method, respectively. In case of Li and Orchard's method, smoothness of edges is good, whereas sharpness is not satisfactory. In Greenspan et al.'s method, resolution is improved around the character region and building boundary, whereas unnecessary changes appear in smooth background regions, thus the reconstructed image looks like a noisy image. Also, overshoots appear near edges, thus the reconstructed image is not visually satisfactory. Similar to Li and Orchard's method, the proposed method gives good edge connectivity than cubic-spline interpolation. Also, the proposed algorithm yields sharper edges than Li and Orchard's method.

Figures 9(a)-9(d) show $340 \times 300$ zoomed images that are portions of the images in Figure 6(c), interpolated by cubicspline interpolation, Li and Orchard's method, Greenspan et al.'s method, and the proposed method, respectively. A woman in Figure 6(c) is a model who sells cosmetics, and 


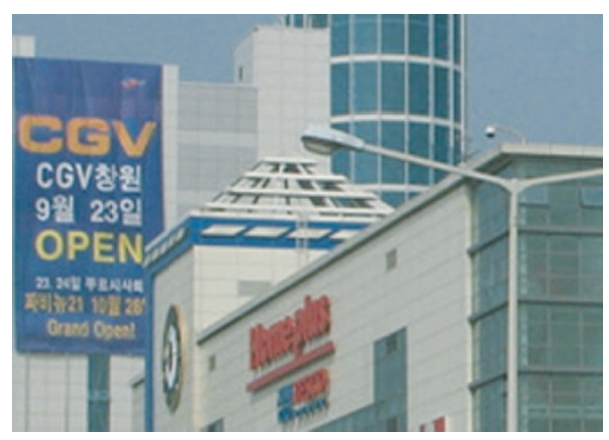

(a)

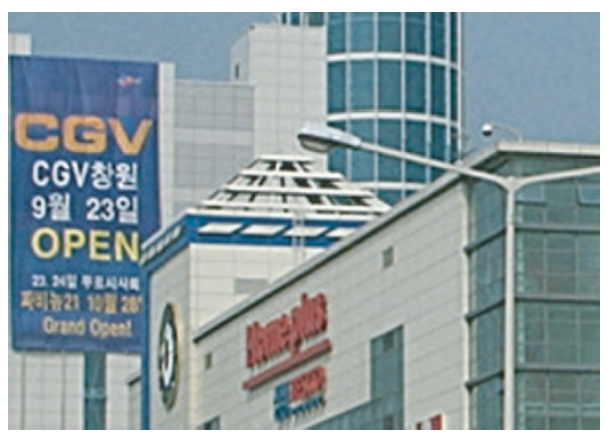

(c)

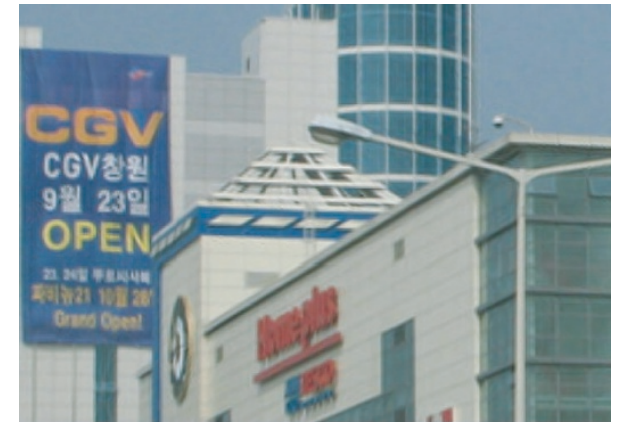

(b)

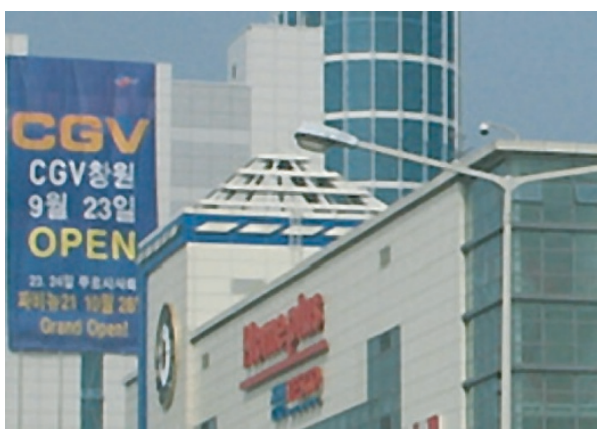

(d)

Figure 8: Comparison of RE results $(1280 \times 960$ Mart image, $200 \%$ zoomed part: $400 \times 280)$ : (a) cubic-spline interpolation, (b) Li and Orchard's method, (c) Greenspan et al.'s method, and (d) proposed algorithm $\left(M_{0}=40, c_{0}=0.0\right)$.

her face can be the region of interest at home shopping. So, $\mathrm{RE}$ of the face region in an image is important. As in the results of the Gate and Mart images, the proposed method shows better results than the conventional methods. Also, Figure 9(e) shows the comparison of interpolated edge profiles along the bold black lines that are superimposed in Figures 9(a)-9(d). Figure 9(e) illustrates that the proposed algorithm yields sharp edge and reduced overshoot artifacts, whereas the other methods show large overshoots or blurring artifacts near edges.

\subsection{Computational load and PSNR comparison}

Table 1 shows performance comparison of each method for the RGB Woman image in terms of the computation time, in which a PC with $2.0 \mathrm{GHz}$ Pentium IV (1 GB RAM) is used. Note that the computation time represents the time taken to interpolate an input image by a factor of two horizontally and vertically. Cubic-spline interpolation cannot effectively produce high-quality images with the smallest amount of computation because of the simplicity of the algorithm. In contrast, Li and Orchard's method produces high-quality images with the highest computational load. In the proposed algorithm, once Gaussian/Laplacian pyramid is generated (most of the time is taken in this processing), final results are generated with the estimated scale constant $\beta$. In the proposed algorithm, the computational cost is reasonable with respect to quality requirement. Also, if we can preestimate an optimal constant $\beta$ using a set of training images, only a single pyramid level $(N=1)$ is required.

Table 2 shows performance comparison of each method for each test image in terms of the PSNR. We acquire downsampled image for each test image using the REDUCE (.) operator (see (1)). Downsampled images are interpolated by four interpolation methods: cubic-spline interpolation method, Greenspan et al.'s method, Li and Orchard's method, and the proposed method. The proposed algorithm is somewhat superior to the three conventional methods in terms of the PSNR, though the PSNR is not an absolute measure for performance comparison of the resolution enhancement region.

\section{CONCLUSIONS}

In this paper, a new RE method for still images is proposed, in which lost HF components are estimated based on the Laplacian pyramid. In predicting the HF image, statistical characteristics of the Laplacian images are utilized, where the normalized histogram of the Laplacian image data is fitted to the Laplacian pdf and the scaling constant $\beta$ is estimated. 


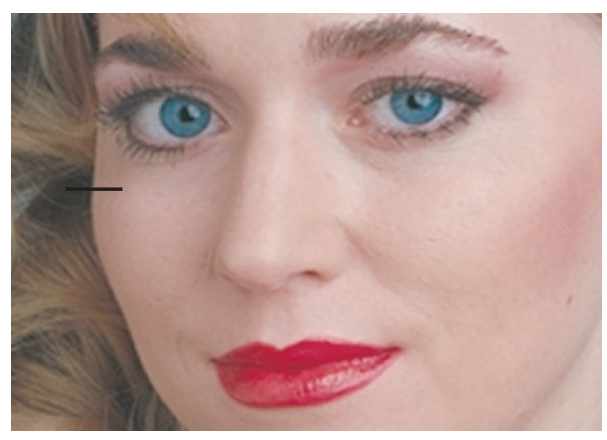

(a)

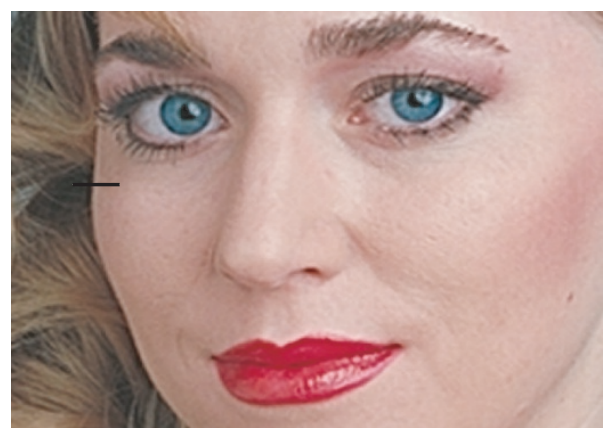

(c)

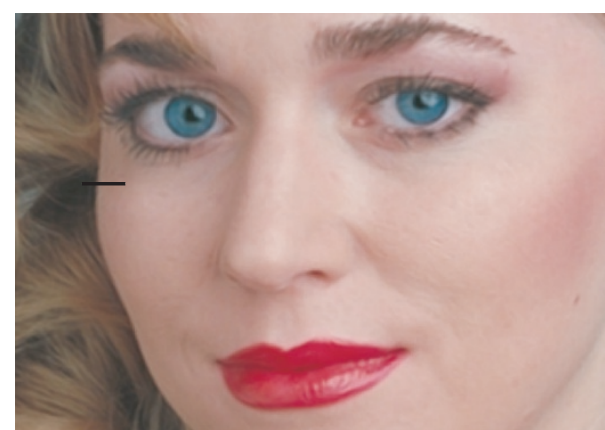

(b)

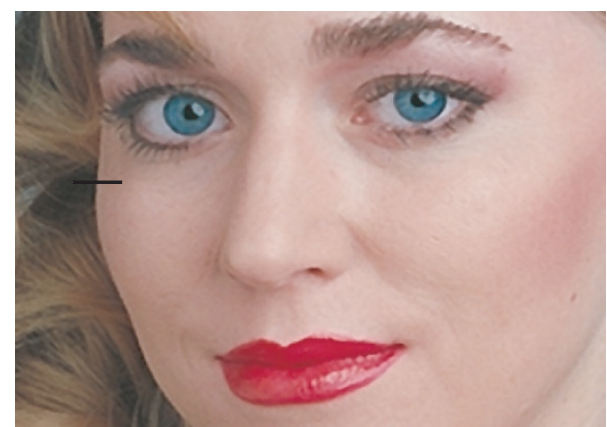

(d)

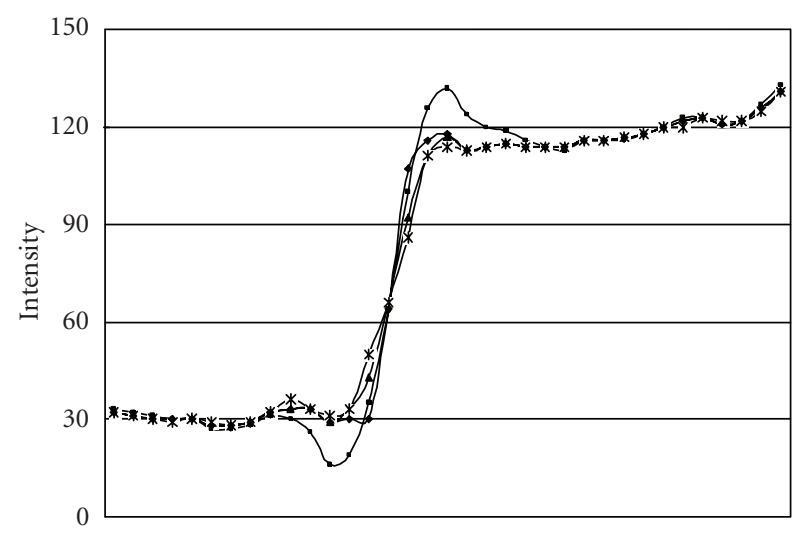

- Cubic-spline interpolation method

-*- Li and Orchard's method

- Greenspan et al.'s method

- Proposed algorithm

(e)

FIGURE 9: Comparison of RE results $(1024 \times 1536$ Woman image, $200 \%$ zoomed part: $340 \times 300)$ : (a) cubic-spline interpolation, (b) Li and Orchard's method, (c) Greenspan et al's method, (d) proposed algorithm $\left(M_{0}=50, c_{0}=0.0\right)$, and (e) comparison of interpolated edge profiles.

A control function as a postprocessing step is employed to suppress small variations in smooth regions and to remove overshoot artifacts in edge regions.

In experiments, three test images are used to show the effectiveness of the proposed algorithm. Comparison of subjective quality of the reconstructed images shows that the proposed method shows better results than the conventional $\mathrm{RE}$ methods in terms of connectivity and sharpness in edge regions and smoothness in uniform regions. Further research will focus on automatic estimation of two parameters $\left(M_{0}\right.$ and $c_{0}$ ) of the control function and on extension of the algorithm for video. 
TABLE 1: Computation time of each RE method.

\begin{tabular}{lccc}
\hline Cubic-spline interpolation & Li and Orchard's method & Greenspan et al.'s method & Proposed method \\
\hline $0.48 \mathrm{~s}$ & $21.33 \mathrm{~s}$ & $3.62 \mathrm{~s}$ \\
\hline \multicolumn{4}{c}{$512 \times 768$ image $\rightarrow 1024 \times 1536$ image } \\
Gaussian/Laplacian pyramid generation: $3.01 \mathrm{~s}$ & \\
\hline
\end{tabular}

TABLE 2: PSNR comparison (dB).

\begin{tabular}{lccc}
\hline & Figure 7 (Gate image) & Figure 8 (Mart image) & Figure 9 (Woman image) \\
\hline Cubic-spline interpolation & 25.20 & 28.12 & 27.80 \\
Greenspan et al.'s method & 25.07 & 28.72 & 26.23 \\
Li and Orchard's method & 27.01 & 29.76 & 31.02 \\
Proposed method & 27.89 & 30.55 & 30.98 \\
\hline
\end{tabular}

\section{ACKNOWLEDGMENT}

This work was supported in part by Samsung Electronics Corporation, Ltd.

\section{REFERENCES}

[1] S. C. Park, M. K. Park, and M. G. Kang, "Super-resolution image reconstruction: a technical overview," IEEE Signal Processing Magazine, vol. 20, no. 3, pp. 21-36, 2003.

[2] A. K. Jain, Fundamentals of Digital Image Processing, PrenticeHall, Englewood Cliffs, NJ, USA, 1989.

[3] R. C. Gonzalez and R. E. Woods, Digital Image Processing, Prentice-Hall, Upper Saddle River, NJ, USA, 2nd edition, 2002.

[4] X. Li and M. T. Orchard, "New edge-directed interpolation," IEEE Transactions on Image Processing, vol. 10, no. 10, pp. 1521-1527, 2001.

[5] A. Biancardi, L. Cinque, and L. Lombardi, "Improvements to image magnification," Pattern Recognition, vol. 35, no. 3, pp. 677-687, 2002.

[6] J.-G. Leu, "Sharpness preserving image enlargement based on a ramp edge model," Pattern Recognition, vol. 34, no. 10, pp. 1927-1938, 2001.

[7] Q. Wang and R. Ward, "A contour-preserving image interpolation method," in Proc. IEEE International Conference on Image Processing (ICIP '03), vol. 3, pp. 673-676, Barcelona, Spain, September 2003.

[8] H. Greenspan, C. H. Anderson, and S. Akber, "Image enhancement by nonlinear extrapolation in frequency space," IEEE Transactions on Image Processing, vol. 9, no. 6, pp. 1035-1048, 2000.

[9] Y. Takahashi and A. Taguchi, "An arbitrary scale image enlargement method with the prediction of high-frequency components," Electronics and Communications in Japan (Part III: Fundamental Electronic Science), vol. 86, no. 8, pp. 41-51, 2003.

[10] W. K. Carey, D. B. Chuang, and S. S. Hemami, "Regularitypreserving image interpolation," IEEE Transactions on Image Processing, vol. 8, no. 9, pp. 1293-1297, 1999.

[11] W. T. Freeman, T. R. Jones, and E. C. Pasztor, "Example-based super-resolution," IEEE Computer Graphics and Applications, vol. 22, no. 2, pp. 56-65, 2002.
[12] J. Sun, N.-N. Zheng, H. Tao, and H.-Y. Shum, "Image hallucination with primal sketch priors," in Proc. IEEE International Conference on Computer Vision and Pattern Recognition (CVPR '03), vol. 2, pp. 729-736, Madison, Wis, USA, June 2003.

[13] P. J. Burt and E. H. Adelson, "The Laplacian pyramid as a compact image code," IEEE Transactions on Communications, vol. 31, no. 4, pp. 532-540, 1983.

[14] G. Ramponi and A. Polesel, "Rational unsharp masking technique," Journal of Electronic Imaging, vol. 7, no. 2, pp. 333-338, 1998.

[15] F. A. Cheikh and M. Gabbouj, "Directional-rational approach for color image enhancement," in Proc. IEEE Int. Symp. Circuits and Systems (ISCAS '00), vol. 3, pp. 563-566, Geneva, Switzerland, May 2000.

Bo-Won Jeon was born in Korea in 1976. He received the B.S. degree in electrical information control engineering from Myongji University in 2002 and the M.S. degree in electronic engineering from Sogang University in 2004. His current research interests are video processing and resolution enhancement.

Rae-Hong Park was born in Seoul, Korea, in 1954. He received the B.S. and M.S. degrees in electronics engineering from Seoul National University, Seoul, Korea, in 1976 and 1979, respectively, and the M.S. and Ph.D. degrees in electrical engineering from Stanford University, Stanford, Calif, USA, in 1981 and 1984, respectively. In 1984, he joined the faculty of the Department of Electronic Engineering, Sogang University,
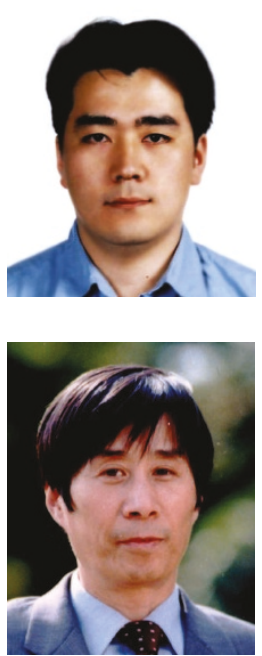

Seoul, Korea, where he is currently a Professor. In 1990, he spent his sabbatical year as a Visiting Associate Professor with the Computer Vision Laboratory, Center for Automation Research, University of Maryland at College Park, USA. His current research interests are video communication, computer vision, and pattern recognition. He served as Editor for the Korea Institute of Telematics and 
Electronics (KITE) Journal of Electronics Engineering from 1995 to 1996 . He was the recipient of a 1990 postdoctoral fellowship presented by the Korea Science and Engineering Foundation (KOSEF), the 1987 Academic Award presented by the KITE, the 2000 Haedong Paper Award presented by the Institute of Electronics Engineers of Korea (IEEK), the 1997 First Sogang Academic Award, and the 1999 Professor Achievement Excellence Award presented by Sogang University.

Seungjoon Yang received the B.S. degree from Seoul National University, Seoul, Korea, in 1990, and the M.S. and Ph.D. degrees from the University of Wisconsin-Madison, USA, in 1993 and 2000, respectively, all in electrical engineering. In 2000, he joined the Digital Media Research and Development Center, Samsung Electronics Corporation, Ltd., Suwon, Korea. His research interests are in image processing, estimation

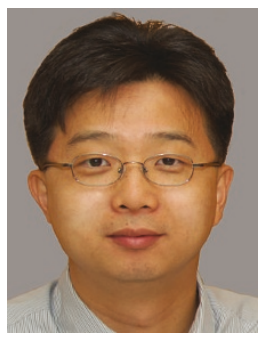
theory, and multirate systems. 